\title{
The Finite Difference Methods for Parabolic Partial Differential Equations
}

\section{Nigatie $Y^{*}$}

Department of Mathematics, College of Natural and Computational Sciences, University of Gondar, Gondar, Ethiopia

\author{
Abstract \\ In this paper finite difference methods will used to solve both one and two dimensional heat equations which are \\ the well-known partial differential equations.
}

Keywords: Parabolic; Heat equation; Finite difference; BenderSchmidt; Crank-Nicolson

\section{Introduction}

\section{Parabolic partial differential equations}

The well-known parabolic partial differential equation is the one dimensional heat conduction equation [1]. The solution of this equation is a function $u(x, t)$ which is defined for values of $x$ from 0 to $l$ and for values of $t$ from 0 to $\infty$ [2-4]. The solution is not defined in a closed domain but advances in an open ended region from initial values, satisfying the prescribed boundary conditions (Figure 1). In general the study of pressure waves in a fluid, propagation of heat and unsteady state problems lead to parabolic type of equations [5].

\section{Solution of one-dimensional Heat conduction equation}

Consider the heat conduction which is of the form [6]

$$
c u_{t}=u_{x x}
$$

where $\mathrm{C}$ is a constant Let the plane $(\underline{x, t})$ be divided in to smaller rectangles by means of the sets of lines [7]

$x=i h, i=0,1,2$.

$t=j k, j=0,1,2$.

Using the finite difference approximations [8-11]

$$
u_{t}=\frac{u_{i, j+1}-u_{i, j}}{k}
$$

and

$$
u_{t t}=\frac{u_{i-1, j}-2 u_{i, j}+u_{i+1, j}}{h^{2}}
$$

Hence the finite difference analogue in eqn. (1) is

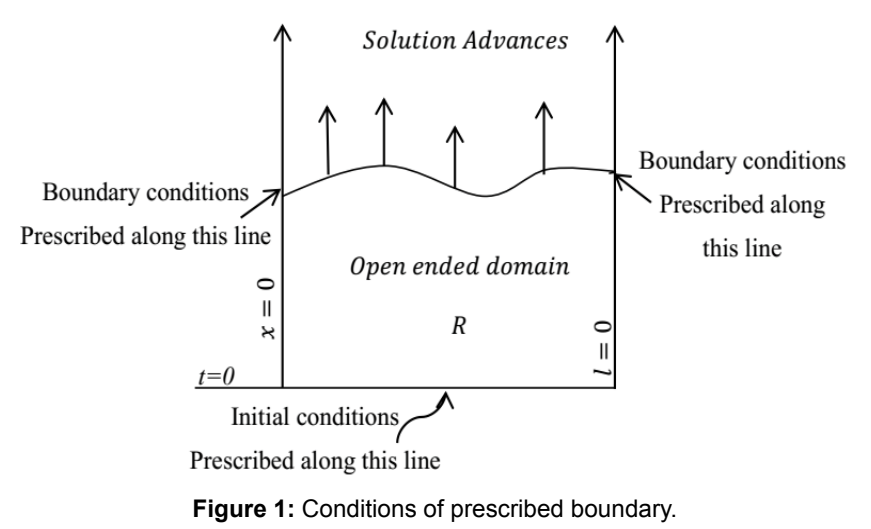

$$
\frac{c}{k}\left(u_{i, j+1}-u_{i, j}\right)=\frac{1}{h^{2}}\left(u_{i-1, j}-2 u_{i, j}+u_{i+1, j}\right)
$$

Which can be rewritten as

$$
u_{i, j+1}=u_{i, j}+\lambda\left(u_{i-1, j}-2 u_{i, j}+u_{i+1, j}\right)
$$

where $\lambda=\frac{k}{c h^{2}}$ this formula expresses the unknown function value at the $(i, j+1)^{\text {th }}$ interior point in terms of the known function values and hence it is called the explicit formula. It can be shown that this formula is valid only [10] for $0<\lambda<\frac{1}{2}$ For $\lambda=\frac{1}{2}$ becomes in eqn. (4)

$$
u_{i, j+1}=\frac{1}{2}\left(u_{i-1, j}+u_{i+1, j}\right)
$$

The above formula in eqn. (5) is called Bender-Schmidt recurrence relation. In formula in eqn. (4), we have used the function values along the $j^{\text {th }}$ row only in the approximation of $u_{x x}$ is replaced by the [11] average of its finite difference approximations on the $j^{\text {th }}$ and $(i, j+1)^{\text {th }}$ rows. Thus

$$
u_{x x}=\frac{1}{2}\left(\frac{u_{i-1, j}-2 u_{i, j}+u_{i+1, j}}{h^{2}}+\frac{u_{i-1, j+1}-2 u_{i, j+1}+u_{i+1, j+1}}{h^{2}}\right)
$$

and hence in eqn. (1) is replaced by

$$
\frac{c}{k}\left(u_{i+1, j}-u_{i, j}\right)=\frac{1}{2 h^{2}}\left(u_{i-1, j}-2 u_{i, j}+u_{i+1, j}+u_{i-1, j+1}-2 u_{i, j+1}+u_{i+1, j+1}\right)
$$

This implies

$$
\left.-\lambda u_{i-1, j+1}\right)+(2+2 \lambda) \mathrm{u}_{i, j+1}-\lambda u_{i+1, j+1}=\lambda u_{i-1, j}+(2-2 \lambda) u_{i, j}+\lambda u_{i+1, j}
$$

where $\lambda=\frac{k}{c h^{2}}$, on the left side in eqn. (6) we have three unknowns and on the right side all the three quantities are known. The above formula in eqn. (6) which is an implicit scheme is called Crank-Nicolson formula and it is convergent for all finite values of $\lambda$. If there are $N$ internal mesh points on each row, then the formula in eqn. (6) gives $N$ simultaneous equations for the $N$ unknowns in terms of the given boundary values.

*Corresponding author: Nigatie Y, Department of Mathematics, College of Natural and Computational Sciences, University of Gondar, Gondar, Ethiopia, Tel: +251913080434; E-mail: abynz09@gmail.com

Received August 18, 2018; Accepted September 12, 2018; Published September 27,2018

Citation: Nigatie Y (2018) The Finite Difference Methods for Parabolic Partia Differential Equations. J Appl Computat Math 7: 418. doi: 10.4172/21689679.1000418

Copyright: (c) 2018 Nigatie Y. This is an open-access article distributed under the terms of the Creative Commons Attribution License, which permits unrestricted use, distribution, and reproduction in any medium, provided the original author and source are credited. 
Similarly the internal mesh points on all rows can be calculated [12].

Example 1: Use the Bender-Schmidt recurrence relation to solve the equation $u_{x x}=2 u_{t}$. With the conditions $u(x, 0)=4 x-x^{2}, u(0, t)=u(4, t)=0$ Solution Taking $h=1$ we obtain [13]

$$
k=\frac{1}{2} h^{2} c=1
$$

Also $u(0,0)=0, u(1,0)=3, u(2,0)=4, u(3,0)=3$ and $u(4,0)=0$. For this the first time step, $k=1$.

For this the first time step, $k=1$.

Using Bender-Schmidt recurrence relation, we obtain

$u_{1,1}=\frac{1}{2}(0+4)=2, u_{2,1}=\frac{1}{2}(3+3)=3, u_{3,1}=\frac{1}{2}(4+0)=2$

For $k=2$, we have

$$
\left.u_{1,2}=\frac{1}{2}(0+3)=1.5, u_{2,2}=\frac{1}{2}(2+2)=2, u_{3},\right)=\frac{1}{2}(3+0)=1.5
$$

For $k=3$ we have

$$
\left.u_{1,3}=\frac{1}{2}(0+2)=1, u_{2,3}=\frac{1}{2}(1.5+1.5)=1.5, u_{3},\right)=\frac{1}{2}(2+0)=1
$$

For $k=4$ we have

$$
u_{1,4}=\frac{1}{2}(0+1.5)=0.75, u_{2}=\frac{1}{2}(1+1)=1, u_{3,4},=\frac{1}{2}(1.5+0)=0.75
$$

Similarly with $k=5$ we obtain

$u_{1,5}=\frac{1}{2}(0+1.0)=0.5, u_{2,5}=\frac{1}{2}(0.75+0.75)=0.75, u_{3,5},=\frac{1}{2}(1+0)=0.5$

The computation can be continued to any number of time steps.

Example 2: Solve the equation $u_{t}=u_{x x}$, subject to the conditions, $u(x, 0)=\sin \pi x, 0 \leq x \leq 1, u(0, t)=u(1, t)=0$.

\section{Using}

(a) Bender-Schmidt Method

(b) Crank-Nicolson Method

Carry out the computations for two levels, taking $h=\frac{1}{3}, k=\frac{1}{36}$

\section{Solution}

Here $\mathrm{c}=1, h=\frac{1}{3}$ and $k=\frac{1}{36}$ so that $\lambda=\frac{k}{\left(c h^{2}\right)}=\frac{1}{4}$

Also $u_{1,0}=\sin \frac{\pi}{3}=\frac{\sqrt{3}}{2}, u_{2,0}=\sin \frac{2 \pi}{3}=\frac{\sqrt{3}}{2}$ and all boundary values are zero in Figure 2.

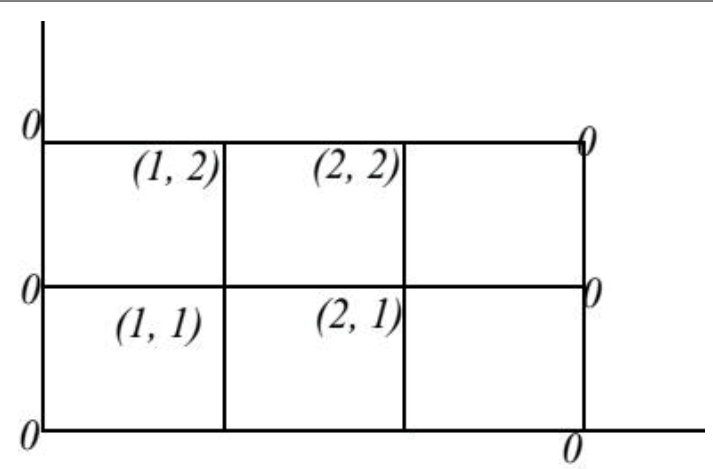

Figure 2: Computations for two levels using Crank-Nicolson method.

\section{(a) Using Bender-Schmidt formula}

Using in eqn. (4) of this section we have $u_{i, j+1}=\lambda u_{i-1}, j+(1-2 \lambda) u_{i, j}+\lambda_{u i+1, j}$

This becomes

$u_{i, j+1}=\frac{1}{4}\left(u_{i-1, j}+2 u_{i, j}+u_{i+1, j}\right)$

For $i=1,2 ; j=0$

$u_{1,1}=\frac{1}{4}\left(u_{0,0}+2 u_{1,0}+u_{2,0}\right)=\frac{1}{4}\left(0+\frac{\sqrt{3}}{2}+\frac{3}{2}\right)=0.65$

$u_{2,1}=\frac{1}{4}\left(u_{1,0}+2 u_{2,0}+u_{3,0}\right)=\frac{1}{4}\left(\frac{\sqrt{3}}{2}+2+\frac{\sqrt{3}}{2}+0\right)=0.65$

For $i=1,2 ; j=1$

$u_{1,2}=\frac{1}{4}\left(u_{0,1}+2 u_{1,1}+u_{2,1}\right)=0.49$
$u_{2,2}=\frac{1}{4}\left(u_{1,1}+2 u_{2,1}+u_{3,1}\right)=0.49$.

(b) By using Crank-Nicolson method

From eqn. (6) above we have

$-\frac{1}{4} u_{i-1, j+1}+\frac{5}{2} u_{i, j+1}-\frac{1}{4} u_{i+1, j+1}=-\frac{1}{4} u_{i-1, j}+\frac{3}{2} u_{i, j}+\frac{1}{4} u_{i+1, j}$

For $i=1,2 ; j=0$

$-u_{0,1}+10 u_{1,1}-u_{2,1}=u_{0,0}+6 u_{1,0}+u_{2,0}$

$\Rightarrow 10 u_{1,1}-u_{2,1}=7 \frac{\sqrt{3}}{2}$

$-u_{1,1}+10 u_{2,1}-u_{3,1}=u_{1,0}+6 u_{2,0}+u_{3,0}$

$\Rightarrow-u_{1,1}+10 u_{2,1}=7 \frac{\sqrt{3}}{2}$

Solving these equations, we find

$u_{1,1}=u_{2,1}=0.67$

For $i=1,2 ; j=1$

$-u_{0,2}+10 u_{1,2}-u_{2,2}=u_{0,1}+6 u_{1,1}+u_{2,1}$

$\Rightarrow 10 u_{1,2}-u_{2,2}=4.69$

$-u_{1,2}+10 u_{2,2}-u_{3,2}=u_{1,1}+6 u_{2,1}+u_{3,1}$

$\Rightarrow-u_{1,2}+10 u_{2,2}=4.69$

Again solving the above two equation we can obtain

$u_{1,2}=u_{2,2}=0.52$.

\section{Solution of two dimensional heat equations}

Two dimensional heat equations is an equation of the form $u_{t}=c^{2}\left(u_{x x}+u_{y y}\right)$.

The method employed for the solution of one dimensional heat equation can be readily extended to the solution two dimensional heat equations in eqn. (7).

Consider a square region $0 \leq x \leq y \leq a$ and assume that $\mathrm{u}$ is known at all points within and on the boundary of this square. 
If $h$ be the step size then a mesh point

$(x, y, t)=(i h, j h, n l)$ may be denoted as $(i, j, n)$.

Replacing the derivatives in eqn. (7) by their finite difference approximations, we get

$$
\begin{aligned}
& \frac{u_{i, j, n+1}-u_{i, j, n}}{l}=\frac{c^{2}}{h^{2}}\left[\left(u_{i-1, j, n}-2 u_{i, j, n}\right)+\left(u_{i, j-1, n}+u_{i, j+1, n}\right)\right] \\
& u_{i, j, n+1}=u_{i, j, n}+\lambda\left(u_{i-1, j, n}+u_{i+1, j, n}+u_{i, j+1, n}+u_{i, j-1, n}-4 u_{i, j, n}\right)
\end{aligned}
$$

Where $\lambda=\frac{l c^{2}}{h^{2}}$. This equation needs the five points available on the $n^{\text {th }}$ plane (Figure 3).

The computation process consists point by point evaluation in the $(n+1)^{\text {th }}$ plane using the points on the nth plane. This method is known as ADE (Alternating Direct Explicit) method.

Example: Solve the equation $u_{t}=u_{x x}+u_{y y}$.

Subject to the initial conditions $u(x, y, 0)=\sin 2 \pi x \sin 2 \pi y, 0 \leq x, y \leq$ 1 and the conditions $u(x, y, t)=0, t \geq 0$, on the boundaries using ADE method $h=\frac{1}{3}$ and $\lambda=\frac{1}{3}$ (Calculate the results for one time level).

Solution: In eqn. (8) becomes

$$
\begin{aligned}
& u_{i, j, n+1}=u_{i, j, n}+\frac{1}{8}\left(u_{i-1, j, n}+u_{i+1, j, n}+u_{i, j+1, n}+u_{i, j-1, n}-4 u_{i, j, n}\right) \\
& u_{i, j, n+1}=\frac{1}{2} u_{i, j, n}+\frac{1}{8}\left(u_{i-1, j, n}+u_{i+1, j, n}+u_{i, j+1, n}+u_{i, j-1, n}\right)(*)
\end{aligned}
$$
below

The mesh points and the computation model is given in Figure 4

At the zeroth level $(n=0)$, the initial and boundary conditions are $u_{i, j, 0}=\sin \frac{2 \pi i}{3} \sin \frac{2 \pi j}{3}$

And

$u_{\mathrm{i}, 0,0}=u_{0, j, 0}+u_{3, j, 0}=u_{\mathrm{i}, 3,0}=0 ; i, j=0,1,2,3$

Now we can calculate the mesh values at the first level.

For $n=0$, from $(*)$ we obtain

$u_{i, j, 1}=\frac{1}{2} u_{i, j, 0}+\frac{1}{8}\left(u_{i-1, j, 0}+u_{i+1, j, 0}+u_{i, j+1,0}+u_{i, j-1,0}\right)(* *)$

(i) Putting $i=j=1$ in $(* *)$ above

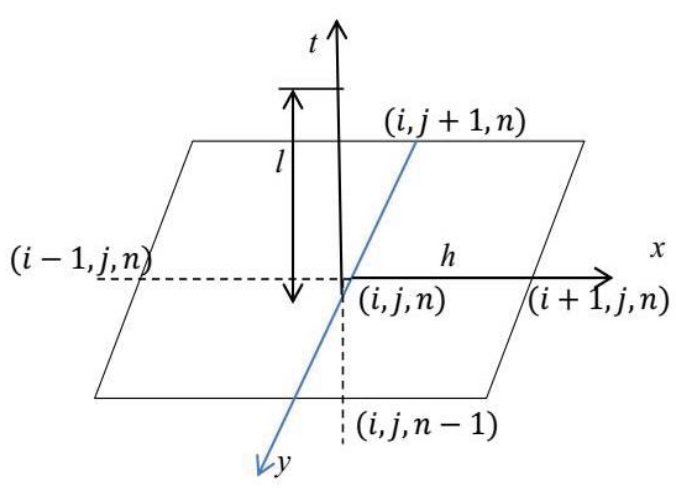

Figure 3: Alternating direct explicit on $(n+1)^{\text {th }}$ plane.

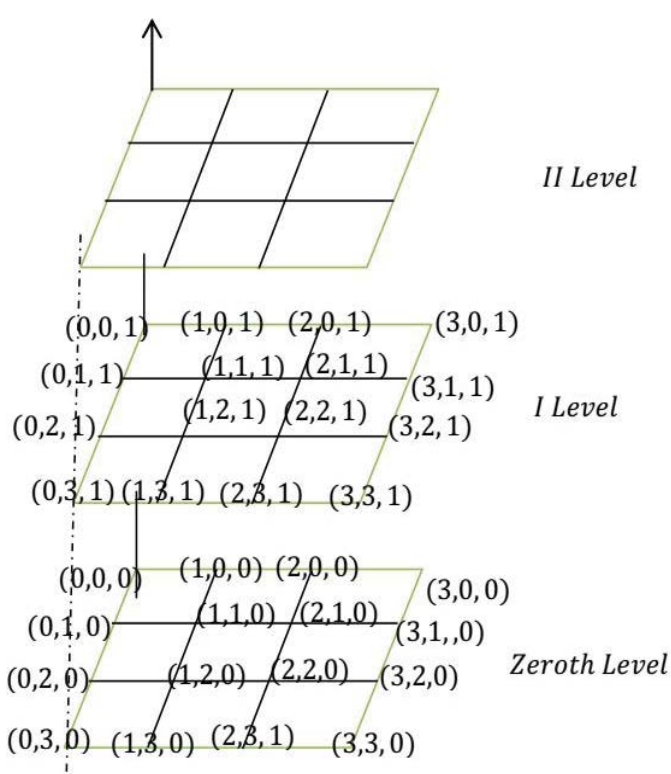

Figure 4: Mesh points and the computation model in zeroth level.

$$
\begin{aligned}
& u_{1,1,1}=\frac{1}{2} u_{1,1,0}+\frac{1}{8}\left(u_{0,1,0}+u_{2,1,0}+u_{2,1,0}+u_{1,0,0}\right) \\
& =\frac{1}{2}\left(\sin \frac{2 \pi}{3}\right)^{2}+\frac{1}{8}\left(0+\sin \frac{4 \pi}{3} \sin \frac{2 \pi}{3}+\sin \frac{2 \pi}{3} \sin \frac{4 \pi}{3}+0\right) \\
& =\frac{3}{8}+\frac{1}{8}\left(-\frac{\sqrt{3}}{2} * \frac{\sqrt{3}}{2}-\frac{\sqrt{3}}{2} * \frac{\sqrt{3}}{2}\right)=\frac{3}{16}
\end{aligned}
$$

(ii) Putting $i=2, j=1$ in $(* *)$ above

$$
\begin{aligned}
& u_{2,1,1}=\frac{1}{2} u_{2,1,0}+\frac{1}{8}\left(u_{0,1,0}+u_{3,1,0}+u_{2,2,0}+u_{2,2,0}\right) \\
& =\frac{1}{2} \sin \frac{4 \pi}{3} \sin \frac{2 \pi}{3}+\frac{1}{8}\left[\left(\sin \frac{2 \pi}{3}\right)^{2}+0+\left(\sin \frac{4 \pi}{3}\right)^{2}+0\right] \\
& =-\frac{1}{2}\left(\frac{\sqrt{3}}{2}\right)^{2}+\frac{1}{8}\left[\left(\frac{\sqrt{3}}{2}\right)^{2}+\left(-\frac{\sqrt{3}}{2}\right)^{2}\right]=-\frac{3}{16}
\end{aligned}
$$

(iii) Putting $i=1, j=2$ in $(* *)$ above

$$
\begin{aligned}
& u_{1,2,1}=\frac{1}{2} u_{1,2,0}+\frac{1}{8}\left(u_{0,2,0}+u_{3,1,0}+u_{1,3,0}+u_{1,1,0}\right) \\
& =\frac{1}{2} \sin \frac{2 \pi}{3} \sin \frac{4 \pi}{3}+\frac{1}{8}\left[0+\left(\sin \frac{4 \pi}{3}\right)^{2}+0+\left(\sin \frac{2 \pi}{3}\right)^{2}\right] \\
& =\frac{3}{8}+\frac{1}{8}\left(\frac{3}{4}+\frac{3}{4}\right)=-\frac{3}{16}
\end{aligned}
$$

(iv) Putting $\mathrm{i}=\mathrm{j}=2$ in $(* *)$ above

$$
\begin{aligned}
& u_{2,2,1}=\frac{1}{2} u_{2,2,0}+\frac{1}{8}\left(u_{1,2,0}+u_{3,2,0}+u_{2,3,0}+u_{2,1,0}\right) \\
& =\frac{1}{2}\left(\sin \frac{4 \pi}{3}\right)^{2}+\frac{1}{8}\left(\sin \frac{2 \pi}{3} \sin \frac{4 \pi}{3}+0+0+\sin \frac{4 \pi}{3} \sin \frac{2 \pi}{3}\right) \\
& =\frac{3}{8}+\frac{1}{8}\left(-\frac{3}{4}-\frac{3}{4}\right)=-\frac{3}{16}
\end{aligned}
$$

Similarly the mesh values at the second and higher levels can be calculated. 
Citation: Nigatie Y (2018) The Finite Difference Methods for Parabolic Partial Differential Equations. J Appl Computat Math 7: 418. doi: 10.4172/21689679.1000418

Page 4 of 4

\section{References}

1. Jaiswal AK, Khandelwal A (2009) A text book of computer based numerical and statistical techniques, p: 618

2. Kusalaas $\mathrm{J}(2005)$ Numerical Methods in Engineering with python.

3. Neumerier A (2001) Introduction to Numerical Analysis. (1stedn.), United Kingdom, Cambridge.

4. Faires, Burde (2000) Numerical Methods (Problems and Solutions). (3rdedn).

5. Jain MK, lyengar SRK, Jain RK (2005) Numerical Methods for Scientific and Engineering Computation.

6. Suli E, Mayers DF (2003) An Introduction to Numerical Analysis. New York, Cambridge.
7. Dahlquist G, Bjorck A (2008) Numerical Methods in Scientific Computing Society for Industrial and Applied Mathematics.

8. Zaliznaik V (2008) Essentials of scientific computing (1stedn.), Numerical Methods in Science and Engineering.

9. lyengar SRK, Jain RK (2009) Numerical Methods, New Age International Publishers, pp:182-184.

10. Scott LR (2011) Numerical Analysis.United States of America, Princeton.

11. Karris ST (2004) Numerical Analysis Using MATLAB and spread sheets. (2ndedn), USA, Orchard.

12. Chapra SC (2007) Applied Numerical Methods with MATLAB, (2ndedn).

13. Karris ST (2004) Numerical Analysis Using MATLAB and spread sheets (2ndedn) USA, Orchard. 